\title{
Correction to: Disruption of endothelial adherens junctions by high glucose is mediated by protein kinase $C$ - $\beta$-dependent vascular endothelial cadherin tyrosine phosphorylation
}

\author{
Mehran Haidari ${ }^{1,2^{*}}$, Wei Zhang ${ }^{2}$, James T. Willerson ${ }^{1,2}$ and Richard A. F. Dixon ${ }^{2}$
}

\section{Correction to: Cardiovasc Diabetol (2014) 13:112 DOI 10.1186/1475-2840-13-112}

This article was unintentionally published twice in this journal, by the same authors. The following should be considered the version of record and used for citation purposes: "Mehran Haidari, Wei Zhang, James T Willerson and Richard AF Dixon, Disruption of endothelial adherens junctions by high glucose is mediated by protein kinase $C$ - $\beta$-dependent vascular endothelial cadherin tyrosine phosphorylation, Cardiovascular Diabetology, Volume 13, Issue 1, doi: 10.1186/1475-2840-13-112“. The duplicate "Mehran Haidari, Wei Zhang, James T Willerson and Richard AF Dixon, Disruption of endothelial adherens junctions by high glucose is mediated by protein kinase $C$ - $\beta$-dependent vascular endothelial cadherin tyrosine phosphorylation, Cardiovascular Diabetology, Volume 13, Issue 1, doi: $10.1186 / 1475-2840-13-105^{\prime \prime}$ is to be ignored. The Publisher apologizes to the readers of the journal for not detecting the duplication during the publication process.

\section{Author details}

1 Department of Internal Medicine, Division of Cardiology, The University of Texas Medical School at Houston, Houston, TX 77030, USA. ${ }^{2}$ Texas Heart Institute at St. Luke's Episcopal Hospital, PO Box 20345 C1000, Houston, TX 77030, USA.
The online version of the original article can be found under doi:10.1186/1475-2840-13-112

\section{Publisher's Note}

Springer Nature remains neutral with regard to jurisdictional claims in published maps and institutional affiliations.

Received: 4 October 2017 Accepted: 4 October 2017

Published online: 20 October 2017

\section{Reference}

1. Haidari M, Zhang W, Willerson JT, Dixon RAF. Disruption of endothelial adherens junctions by high glucose is mediated by protein kinase C- $\beta$-dependent vascular endothelial cadherin tyrosine phosphorylation. Cardiovasc Diabetol. 2014;13(1):112. doi:10.1186/1475-2840-13-112. 